\title{
Implementation of the Signs of Suicide Prevention Program with 9th Grade Students in a Public School Setting
}

\author{
Jessica Tennant
}

Follow this and additional works at: https://researchrepository.wvu.edu/etd

\section{Recommended Citation}

Tennant, Jessica, "Implementation of the Signs of Suicide Prevention Program with 9th Grade Students in a Public School Setting" (2017). Graduate Theses, Dissertations, and Problem Reports. 6782.

https://researchrepository.wvu.edu/etd/6782

This Dissertation is protected by copyright and/or related rights. It has been brought to you by the The Research Repository @ WVU with permission from the rights-holder(s). You are free to use this Dissertation in any way that is permitted by the copyright and related rights legislation that applies to your use. For other uses you must obtain permission from the rights-holder(s) directly, unless additional rights are indicated by a Creative Commons license in the record and/ or on the work itself. This Dissertation has been accepted for inclusion in WVU Graduate Theses, Dissertations, and Problem Reports collection by an authorized administrator of The Research Repository @ WVU.

For more information, please contact researchrepository@mail.wvu.edu. 


\title{
Implementation of the Signs of Suicide Prevention Program with $9^{\text {th }}$ grade students in a Public School Setting
}

\author{
Jessica Tennant, DNP, CRNP \\ Dissertation submitted \\ to the School of Nursing \\ at West Virginia University \\ in partial fulfillment of the requirements for the degree of \\ Doctorate of Practice in \\ Nursing \\ Marilyn Smith, Ph.D., PMHNP-BC, APRN, Committee Chair \\ Mary Jane Smith, Ph.D, RN, FAAN, Committee Member \\ Susan Newfield, Ph.D, RN, PMHCNS-BC, Committee Member
}

Department of Nursing

Morgantown, West Virginia

2017

Keywords: suicide prevention, adolescent depression, Signs of Suicide Prevention Program Copyright 2017 Jessica R. Tennant, DNP, CRNP 


\title{
ABSTRACT \\ Implementation of the Signs of Suicide Prevention Program with $9^{\text {th }}$ grade students in a Public School Setting
}

\author{
Jessica R. Tennant, DNP, CRNP
}

This quality improvement project examined the effectiveness of the use of the Signs of Suicide Prevention Program for educating $9^{\text {th }}$ grade students. Pre-tests and post-tests were administered to twelve participating students which focused on knowledge about depression, suicide and suicide prevention. A t-test was performed to evaluate for a difference in pre-test and post-test scores. A single open-ended question was included on the post-test, "Please write 2 things you could do to prevent suicide?" Results were found to be statistically significant $(\mathrm{t}(11)=4.432$, $\mathrm{p}=0.01), 95 \%$ confidence interval $(-2.49,-0.84)$. The overall mean difference was -1.67 . Additional descriptive data was collected through group discussion on "What kind of changes may you see in a friend that could be a potential sign of depression?" Based on a review of collected data, the Signs of Suicide Prevention Program demonstrated the potential benefits of a public school system establishing a role in the mental health education and suicide prevention effort. 


\section{TABLE OF CONTENTS}

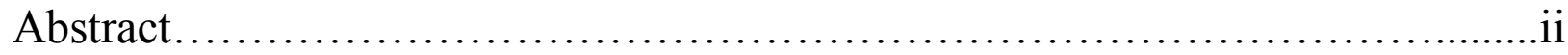

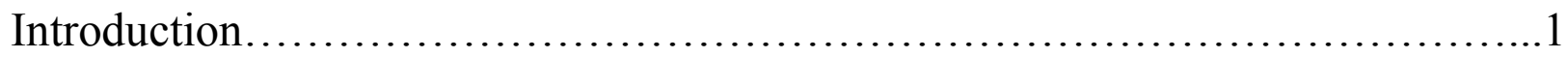

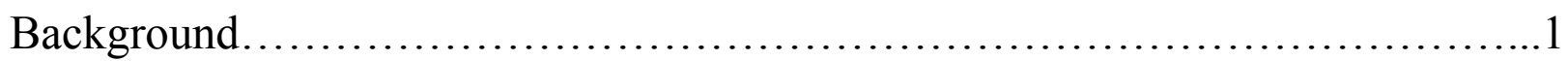

Evidence of Proposed Change.........................................4

Problem Statement...................................................

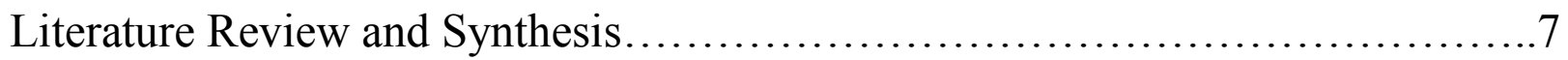

Clinical Practice Guidelines and Recommendations...................... 8

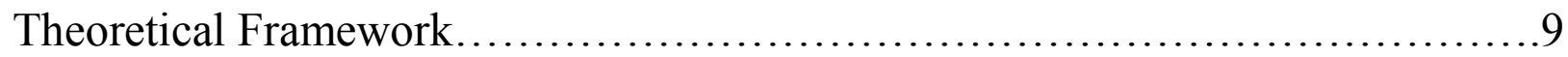

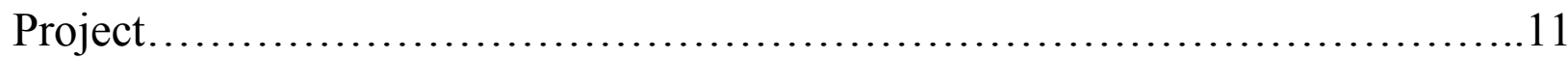

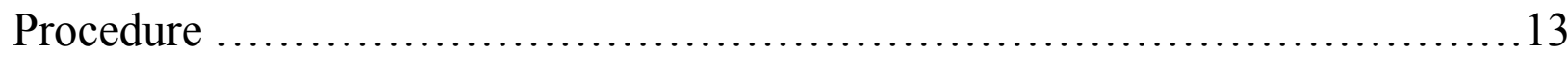

Project Objectives................................................. 15

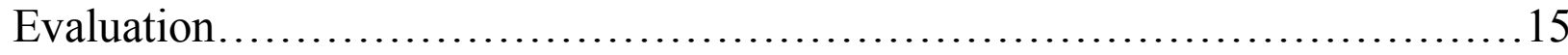

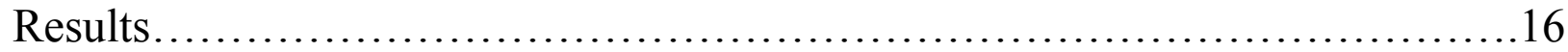

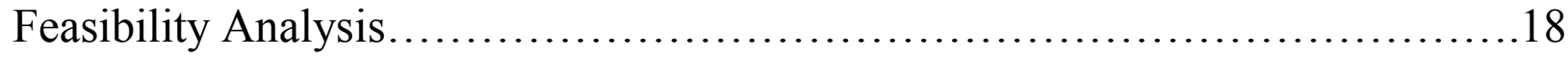

Identification of Quality Improvement Project Resources....................19

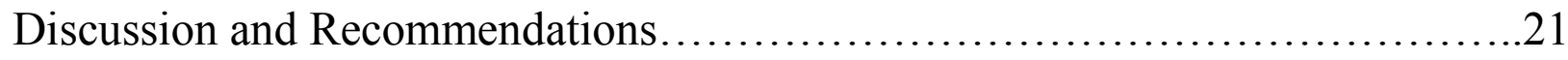


Implementation of the Signs of Suicide Prevention Program with $9^{\text {th }}$ grade students in a Public School Setting

\section{Introduction}

Suicide is the second leading cause of death in children and adolescents ages 11-18 (Centers for Disease Control, 2014). Fifteen percent of teens have seriously considered attempting suicide (Centers for Disease Control, 2014). Adolescents are a vulnerable population who deserve a tremendous amount of attention and care due to impulsivity related to their developmental age and stage which increases risk for suicide. Despite the American Academy of Pediatrics (n.d.) recommendation of yearly wellness exams for this age group, only 6 in 10 actually report seeing a Primary Care Provider on a yearly basis. Additionally, the United States Preventative Task Force Service (USPTFS) recommends yearly mental health screening in the primary care setting for persons age 12-17; however, Healthy People 2020 reports that only $1.9 \%$ of PCP offices currently participate in this practice (2016). It seems pertinent to consider the role the public school system can play in the education and prevention of suicide due to the multitude of stressors that adolescents face daily in home, school and their societal circumstances.

\section{Background}

\section{Challenges}

Erikson's Stage of Psychosocial Development, Identity vs. Role Confusion, which begins at age 12 (McLeod, 2013) creates significant challenges as it relates to adolescent suicide. During this stage, children begin to look to the future and ultimately want to feel a sense of belonging (McLeod, 2013). Adolescents re-examine their identity and try to establish who they are (McLeod, 2013). Identity crisis or role confusion can occur, which may lead to experimentation 
with different lifestyles, feelings of pressure, negative identity, rebellion and unhappiness (McLeod, 2013).

A challenge related to adolescent suicide is that teachers and staff may believe a student who is at-risk for suicide only exhibits certain behaviors such as absenteeism, or acting out in class. However, there is a wide range of signs or symptoms that may be exhibited in a person with depression or thoughts of suicide. Some signs of depression may be assumed to be "typical teenage behavior" or "hormonal mood swings" (Anxiety and Depression Association of America, 2016). Failure to properly evaluate these symptoms may lead to further suicide attempts or completed suicide.

The stigmatization of mental health services is identified by adolescents as the most significant challenge to the receipt of mental health care (Chandra \& Minkovitz, 2007). Expected negative responses from family members, peers and school staff toward mental health careseeking are major barriers to address mental health concerns for adolescents' (Chandra \& Minkovitz, 2007). Unmet mental health needs among adolescents are further concerns for suicide in adolescents.

\section{Problems}

The incessant use of social media has magnified the platform for bullying and now cyberbullying, both contributing factors to attempted and completed suicide in adolescents. Near forty percent of students report being victims of bullying (NoBullying.com, 2015). Social determinants of health encompass multiple areas which can both positively or negatively affect the mental well-being of a child. These determinants such as; race, gender, income level, geographic location, family dynamic, social support, school conditions, family history and 
community interaction (Healthy People 2020, 2016) can be negative risk factors for suicide and suicide attempts. Alcohol and substance abuse are additional concerns for this vulnerable population since symptoms of substance abuse often erupt by age 15 (Healthy People 2020, 2016). Substance abuse disorders can often be linked to untreated mental health disorders such as depression (Healthy People 2020, 2016), a leading cause of suicide.

\section{Situations}

Significant life events that alter an adolescent's situation can contribute to suicide including; death of a loved one, substance abuse or altered family dynamic (Bolognini, Laget, \& Halfon, 2003). Further, some negative life events (NLE) such as a relationship break up or family argument are often identified as having occurred within 24 or 48 hours of a suicide attempt (Bagge, Glenn \& Lee, 2013). Comorbid conditions such as depression and anxiety increase the risk for suicide in this population as well as prior traumatic experiences or abuse. (Bolognini, Laget, \& Halfon, 2003).

\section{Prevalance}

Suicide is an important public health problem for adolescents all around the world (Kennebeck \& Bonin, 2016). Between the 1960s-1990s the suicide rate doubled in those age 1519 and tripled in ages 10-14 (Kennebeck \& Bonin, 2016). Suicide rates decreased in the early 2000s then increased again (Kennebeck \& Bonin, 2016). While the exact cause is unknown multiple factors aside from increased reporting have been identified, such as; increased rates of drug and alcohol abuse, depression, family and social disorganization and access to firearms (Kennebeck \& Bonin, 2016). In 2009, adolescent suicide accounted for $14 \%$ of total deaths among 15-19 year olds; 1,922 completed suicides in a single year (Kennebeck \& Bonin, 2016). 
From 2009-2011, in Pennsylvania alone, there were 138 suicides among children 10-17 years of age (The Bureau of Family Health, 2014). While overall rates per 100,000 are 6.0 and 6.1 respectively for Pennsylvania when compared to the United States, the death rate of black children by suicide in Pennsylvania is double that of the national rate (The Bureau of Family Health, 2014).

The most common condition associated with suicide attempts and completed suicide is depression. According to statistics published by Mental Health America, 9.86\% of youth, age 1217, report suffering from at least one major depression episode in the past year (2016). In total, 18 states, including Pennsylvania, experienced a significant rate increase of major depressive episodes among youth (Mental Health America, 2016). Also of note, 7\% of high school students have attempted to take their own life and nearly $15 \%$ have seriously considered suicide (HealthyPeople2020, 2016). Suicide a leading cause of death in persons age 15-24, accounts for 5,000 deaths per year (Mental Health America, 2016).

\section{Evidence of Proposed Change}

Although childhood is the most important time to promote healthy development (Jacobs, 2016), a persistent gap exists between mental health needs of children and available services (Weist, et. al., 2007). School-based mental health programs services focus primarily on students who have special learning needs identified (Weist, et. al., 2007). Eighty percent of youth with mental illness are not identified or not receiving services (Jacobs, 2016). Over $90 \%$ of those who complete suicide have a diagnosable mental health disorder at time of death (Jacobs, 2016). The most prevalent risk factors for suicide are depression, substance abuse and previous attempts (Jacobs, 2016). Both prevention and early intervention efforts targeted to youth can be efficacious in reducing the detriment untreated mental illness has on school performance, social 
well-being and welfare that carries into adulthood (American Academy of Pediatrics, 2010). Many adult mental health disorders begin in childhood and the single greatest risk factor for adult suicide is prior suicide attempt (Jacobs, 2016).

\section{Opportunities}

Some of the previously presented challenges also serve as opportunities in response to suicide. Because adolescents are in a transitional phase, it presents a great opportunity to work toward overcoming stigma in mental health. As adolescents are learning about their own identity and society, positive representations of treating mental health conditions in the same way as medical conditions can be taught.

\section{Problem Statement}

According to the Centers for Disease Control and Prevention (2016), suicide is the $2^{\text {nd }}$ leading cause of death for children and adolescents age 11-18 in the United States. Nearly 1 in 10, age 12-17 will suffer from at least one major depressive episode in the next year. Depression has been linked to poor school performance, substance use, running away, and feelings of worthlessness, hopelessness and suicide. The implementation of a suicide prevention education within the public school system will allow better access to the population of adolescents in greatest need. The Signs of Suicide Prevention Program addresses many of the age-related concerns and provides education to students to develop personal coping skills (Jacobs, 2015). Additional information is discussed to aid in identification of at-risk students including proper ways to respond through the ACT algorithm which is Acknowledge, Care, Tell (Jacobs, 2015). 


\section{Purpose}

The purpose of this project was to implement the Signs of Suicide Prevention Program in a ninth grade public school setting. This program was aimed at providing suicide education to those students with consent in ninth grade within a public school in southwestern Pennsylvania. The Signs of Suicide Program is an evidence-based education program aimed at decreasing suicide and suicide attempts, as well as to increase help-seeking on behalf of self and for a friend (Jacobs, 2015). Education is aimed at fostering adolescent understanding of mental health illness as treatable conditions in the same fashion as physical conditions (Jacobs, 2015). The implementation of this education program serves as a significant quality improvement when compared to the mental health or suicide prevention education that does not currently exist in the curriculum. This quality improvement project provides a comprehensive process designed to educate students about suicidality and help-seeking methods for the purpose of improved knowledge and the self-help tools vital for decreasing suicide attempts and completions in the adolescent population.

\section{Significance of Project}

The change project is designed to provide suicide prevention education to a single grade of students at a single public school at time of inception. However, the program is designed for expansion and implementation on a yearly basis throughout adolescence in the public school setting. By educating students yearly there is an increase in the likelihood of suicide prevention and will provide adolescents with help-seeking techniques and proper response behaviors for friends in need. Schools are in a great position to render accessible services to youth and reduce the stigma associated with seeking help along the way (Husky, et. al., 2011). 


\section{Literature Review and Synthesis}

Three randomized control trials were included in this literature review and synthesis following an extensive literature search on the implementation of the Signs of Suicide Prevention Program in the public school setting. One study which included the education intervention presentation to 2100 students over five high schools in Georgia and Connecticut utilized selfadministered questionnaires 3-months after program implementation for the basis of outcome measures (Aseltine \& DeMartino, 2004). Data were evaluated for 3 specific outcome categories including, self-reported suicide attempts, knowledge and attitudes about depression and suicide, and help-seeking behavior (Aseltine \& DeMartino, 2004). Results showed statistically significantly reduced rates of self-reported suicide attempts and efficacy in increasing student knowledge and attitudes toward depression and suicide (Aseltine \& DeMartino, 2004). In contrast; however, no significant effects on suicidal ideation and help-seeking behaviors were identified (Aseltine \& DeMartino, 2004).

In 2007, a replicated study was performed on an extended student population to include 4133 students in 9 high schools in Georgia, Connecticut and Massachusetts (Aseltine, James, Schilling \& Glanovsky, 2007). Self-administered questionnaires were completed by both the intervention and control groups 3 months after program implementation to evaluate the same objectives identified in the Year 1 data study by Aseltine \& DeMartino (2004). This extension study confirmed the preliminary results of the first study and found that students in the intervention group reported statistically significant lower rates of suicide attempts, greater knowledge and more adaptive attitudes about depression and suicide (Aseltine, James, Schilling \& Glanovsky, 2007). 
Schilling, Aseltine \& James (2015), further replicated and extended the previous studies through a more rigorous pre-test post-test randomized control design than was previously used in prior evaluation studies of the Signs of Suicide Prevention Program among high school students. Sixteen technical high schools in Connecticut were included in the study with a total of 1575 ninth-grade student participants who were granted parental permission (Schilling, Aseltine \& James, 2015). This evaluation focused on two outcome categories (1) self-reported suicidal ideation, suicide planning and suicide attempts and (2) knowledge and attitudes about depression and suicide (Schilling, Aseltine \& James, 2015). As demonstrated in previous evaluations, participation in the Signs of Suicide Prevention Program was associated with lower rates of suicide attempts, three-months following program implementation (Schilling, Aseltine \& James, 2015). The program was associated with increased student knowledge and favorable attitudes toward help-seeking behaviors or helping a friend with suicidal thoughts or depression (Schilling, Aseltine \& James, 2015). Again; however, there was no improvement in rate of suicidal ideation as noted in the previous studies (Schilling, Aseltine \& James, 2015).

\section{Clinical Practice Guidelines and Recommendations}

The U.S. Preventive Services Task Force (USPSTF) (2014), recommends that pediatricians ask questions about mood disorders, sexual orientation, suicidal thoughts and other risks factors associated with suicide during routine office visits. However, current evidence to assess benefit and harm balance is insufficient for evaluating suicide risks for adolescents in primary care practice (USPSTF, 2014). The USPSTF, reports that cost for assessing suicide risk is minimal and as suicide is the second leading cause of death in this age group, those identified as having risk factors for suicide should be identified and further evaluated for prevention of suicide (USPSTF, 2014). 


\section{Theoretical Framework}

The Social Learning Theory explains human behavior through reciprocal interaction on a continuous basis between cognitive, behavioral and environmental influences (Learning Theories, 2015). Necessary conditions for effective modeling and reciprocal determinism are the two key concepts of the Social Learning Theory (Learning Theories, 2015). The concept of necessary conditions for effective modeling is met through the attainment of attention, retention, reproduction and motivation (Learning Theories, 2015). These conditions may be affected either positively or negatively by level of arousal, perception, sensory capabilities or motivation to imitate for example (Learning Theories, 2015).

Necessary conditions for effective modeling were achieved through the viewing of the 22minute educational video and the environment where it was watched. Learning through effective modeling occurred when the four necessary conditions were met. Attention was heightened and learning improved when conditions were positively affected. The intervention took place in a classroom setting within a small group to decrease outside stimulus and provide a quiet structured environment for learning. Retention was obtained using the visual images displayed in the video to depict the association between the explained concepts and actual observed behaviors. Reproducing explained concepts from the video and the understanding of potential positive outcomes furthered learning by satisfying the final two conditions; reproduction and motivation. Learning occurred through the viewing of this educational film and it is hoped that learning can lead to future modeling of behavior.

Reciprocal Determinism, the second concept, is the term Bandura used to describe his belief that the world and a person's behavior cause each other, while contrastingly behaviorism states that one's environment causes one's behavior (Learning Theories, 2015). Bandura later 
considered that personality is an interaction between three components; behavior, environment and psychological processes (Learning Theories, 2015).

Once the educational video viewing was completed by the student group a brief 10 -minute guided discussion took place with the participants. The focus of the discussion was placed on identifying potential signs of depression or suicide through peer-led discussion and actions. The group was divided into smaller groups of 3 students per group and given a sheet of paper and pen for the group to record their answers. The following open-ended question was posed: "What kind of changes may you see in a friend that could be a potential sign of depression?" Group members had the opportunity to collectively share observed or perceived changes that were identifiable. Through the open discussion students were able to consider one another's responses broadening their own understanding of the signs of depression.

Necessary conditions for effective modeling and reciprocal determinism are two inter-related concepts utilized in the educational intervention. Consideration for the environment, promoting attention, reproduction and providing means for motivation for change were achieved by the viewing the educational film. The group discussion facilitated reciprocal determinism through the sharing of thoughts and ideas amongst peers to further understanding and broaden knowledge of the presented education. Learning was achieved through the relationship between both necessary conditions for effective modeling and reciprocal determinism. First, the conditions for effective modeling were represented through the film viewing within the stated environment, and then was depicted through reciprocal determinism with active discussion among peers leading to learning. 


\section{Model}

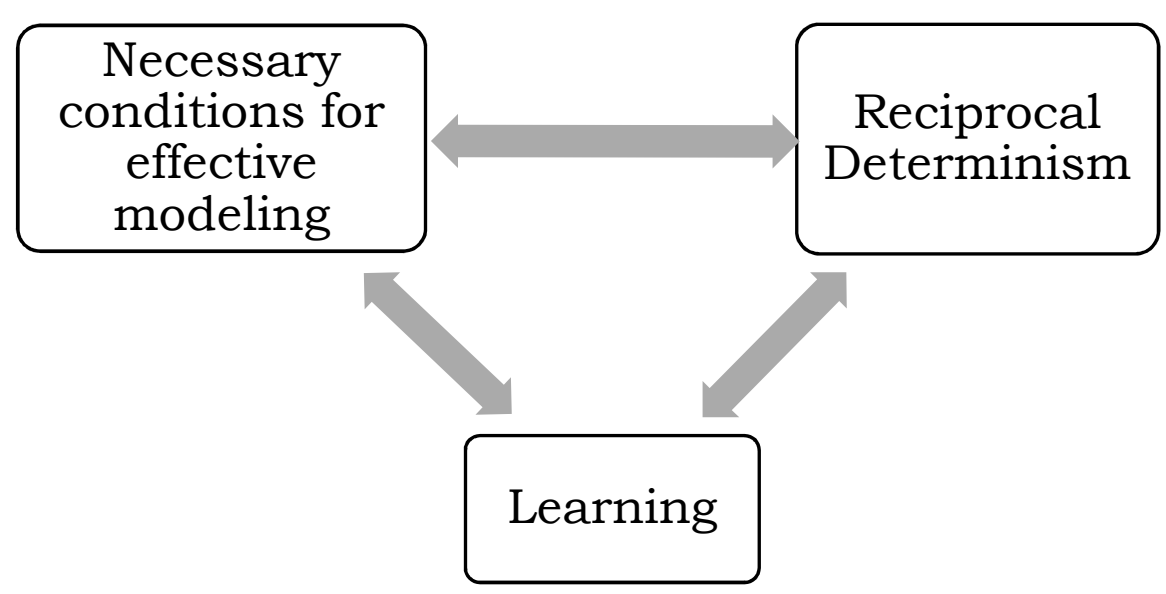

The Signs of Suicide Prevention Program fosters a better understanding of the link between mental illness and physical illness, both require treatment. Adaptive attitudes presented in the intervention aim to reduce the stigma associated with mental illness. Through the knowledge gained by this education intervention, it is hoped that students will be encouraged to utilize helpseeking when in need and empowered to intervene on behalf of a friend who exhibits symptoms of depression/suicidality. By educating students and increasing their self-efficacy, it is hoped that participants gain skills to promote health, both physical and mental.

\section{Project}

Approval: The initiation of the proposed project began with the approval from school administrators and school board members for the implementation of the educational intervention as part of a pilot study. (See Appendix A)

Education: Teachers and other staff with direct contact with the students were shown the Adult Training Video which was part of the Signs of Suicide Prevention Program (Jacobs, 2015). The Adult Training Video is 28-minutes in length and presented a synopsis of Signs of Suicide Prevention Program Student Video (Jacobs, 2015). 
Strategy for Implementation: Following the viewing of the film by teachers and staff, available dates for implementation to all ninth grade students were identified. Further implementation details were identified such as which classroom would be used and the process by which students were consented. The education intervention was given to students during a single class period on the pre-determined date.

Parental Consent: As guided by the Institutional Review Board comprehensive parental consent forms (Appendix B) were distributed to all 96 students enrolled in the $9^{\text {th }}$ grade of the given school.

Student Assent/Intervention: Twelve students (12\%) with completed and returned parental consent forms were included in the intervention. At the pre-determined class period the student assent (Appendix C) was obtained, all 12 students assented to participate. The pre-test (Appendix D) was then given for completion prior to education intervention. Following completion of the pre-test, Signs of Suicide Prevention Education video (22-minutes in length) was shown the group of students. The intervention video educated students based on the ACT: Acknowledge, Care, Tell acronym as it relates to suicide prevention and the recognition of possible signs of depression (Jacobs, 2015). This evidenced-based education program utilized short vignettes to depict real-life scenarios and how to respond to them if faced in the future (Jacobs, 2015). Upon completion of the viewing of the educational film the students were lead in a 10- minute discussion based on a key concept of the film; what changes you may see in a friend that is a potential sign of depression. Following the group discussion the post-test (Appendix E) for knowledge was given to each student for completion. In addition to the post-test, students were given a brief form to complete which offered the student the opportunity to choose if they would or would not like to speak with someone regarding concerns about themselves or a friend. This is 
called a Student Response Card (Appendix F) and each student circled their chosen response and submitted the form prior to exiting the classroom for review.

Review of Results: Following completion of all pre-test/post-test questionnaires scoring was completed and the data were evaluated for effectiveness of education program.

Follow-up: One student requested to speak to someone about self or a friend via the Student Response Card. This card was submitted to the on-site counselor who was present for the intervention. The counselor met with the guidance counselor to determine what if any services the child was receiving and set up a meeting time with that student later the same day.

Design: For the purposes of the proposed project where the intervention consisted of the implementation of Signs of Suicide Prevention Program to $9^{\text {th }}$ grade students within a single public school; the appropriate design was a one group pre/post-test evaluation study. A t-test to analyze pre-test scoring to post-test scoring was used. The 2 additional open ended responses given on the post-test were evaluated for descriptive data:

Please write 2 things you see yourself doing to prevent suicide.

1. 2.

\section{Procedure}

1. Distributed a letter of explanation and parental consent to each ninth grade student's parent/guardian within the participating public school and allowed 14 days to obtain returned letters of consent.

2. Gathered students with signed parental consents in classroom. 
3. Distributed student assent forms, explained content contained on the assent and gave instructions on completion. Assents were completed by each student for participation in the intervention.

4. Provided each participant a pre-test with a number written on both the top and a tab on the bottom of the test with the same number. Verbally instructed the group of students to remove the tab on bottom of test with the number on it and place in on their desks. It was explained that they would later be placing the numbered tab on top of their post-test following the intervention and discussion.

5. Upon completion, pre-tests were collected.

6. Prior to showing the video, announced that any student who wishes to leave at any point during the video they may do so and will be accompanied to a safe place with a staff member.

7. The 22-minute educational film was shown to the classroom of participants.

8. Divided participants into groups of 3 students per group.

9. Led a brief 10-minute discussion guided on principle concepts of the film using the following scripted question:

a. What kind of changes may you see in a friend that could be a potential sign of depression?

10. Distributed the post-tests and reminded participants to transfer the numbered tab from their desk to the bottom of the post-test form.

11. Upon completion, post-tests were collected from participants.

12. Advised students that outside personnel were made available to any student who would like to speak someone about a personal concern or that of a friend. 
a. A Mental Health Counselor from the C.H.I.L.L. (Counseling, Helping, Intervention, Listening, Link) Program was available and on-site for the duration of the study.

13. Instructed students to complete and submit the Student Response Card prior to exiting the classroom.

14. One Student Response Card with the "would like to speak with someone" option chosen was identified upon review and was immediately given to the C.H.I.L.L. Counselor for further coordination with school guidance counselor and follow-up in a discrete manner using pre-existing school policies.

\section{Project Objectives}

Objective \#1: There will be a difference between pre-test and post-test scores.

As part of the education intervention student knowledge on suicide and suicide prevention was tested via a 12-item TRUE/FALSE pre-test completed by each participating student. Following the educational DVD and group discussion, students completed a 12 -item TRUE/FALSE post-test to evaluate the knowledge gained through the educational program as well as complete a 2 response open-ended question for individual response. The pre-test and post-test were comprised of the same 12 questions. The post-test included an open-ended question, "Please write 2 things you see yourself doing to prevent suicide?"

\section{Evaluation Plan}

Objective \#1. Prior to implementation of the education portion of the intervention, students were asked to complete a pre-test which examined the knowledge and understanding of suicide and suicide prevention concepts. Following the educational program and 10minute group discussion, students completed a post-test which knowledge and understanding 
of suicide and suicide prevention concepts were determined. The pre-test/post-test instruments specific to the Signs of Suicide Prevention Program were utilized to examine pre-test/post-test knowledge for this objective. Descriptive statistics in the form of a simple ttest was used to evaluate pre-test/post-test data.

Reponses received in the 2-part open-ended question following the post-test were described for additional data on potential actions for preventing suicide as described by participants. A deductive approach was utilized to analyze participant responses. The review focused on identifying themes present within data. Further evaluation focused on perceived learning by participants through the themes expressed in their written responses. Additional potential for application of knowledge gained from education intervention was identified as a result of the descriptive data collected and analyzed.

\section{Results}

Quantitative. A paired t-test was used to compare the change in each of the individual test scores before and after the intervention, then a comparison of the overall 'pre' and 'post' group scores were performed. From this, a t-test statistic $=4.432$, with degrees of freedom (11), and a $p$-value $=0.01$. The $95 \%$ confidence interval is $(-2.49,-0.84)$. This indicated there was a statistically significant difference between pre and post test scores for this group, with an overall mean difference of -1.67. On average for this group, the pre-test scores were 1.67 points lower than the post-test scores. The mean pre-test score was $79.25 \%$. The mean posttest score was $93.16 \%$. Therefore, objective \#1 was met.

Descriptive. Upon review of participant group responses to the discussion question, "What kind of changes may you see in a friend that could be a potential sign of depression?", 
ten main themes were identified including; (1) distancing from friend, (2) quitting activities, (3) loss of happiness, (4) talk about suicide/depression, (5) grades dropping, (6) drinking/drugs, (7) skipping school, (8) lying to parents and friends, (9) feeling sad/withdrawn and (10) self-harm. Figure 1 demonstrates the frequency which groups responses identified with each of the ten main themes.

Figure 1. Participant Group Responses

\begin{tabular}{|c|c|c|c|c|}
\hline GROUP: & $\# 1$ & $\# 2$ & $\# \mathbf{3}$ & $\# 4$ \\
\hline Distancing from a friend & $\mathrm{X}$ & $\mathrm{X}$ & $\mathrm{X}$ & $\mathrm{X}$ \\
\hline Quitting activities & $\mathrm{X}$ & - & $\mathrm{X}$ & $\mathrm{X}$ \\
\hline Loss of happiness & $\mathrm{X}$ & $\mathrm{X}$ & $\mathrm{X}$ & $\mathrm{X}$ \\
\hline $\begin{array}{r}\text { Talk about } \\
\text { suicide/depression }\end{array}$ & $\mathrm{X}$ & $\mathrm{X}$ & $\mathrm{X}$ & - \\
\hline Grades dropping & $\mathrm{X}$ & $\mathrm{X}$ & - & - \\
\hline Drinking/drugs & $\mathrm{X}$ & $\mathrm{X}$ & $\mathrm{X}$ & $\mathrm{X}$ \\
\hline Skipping school & $\mathrm{X}$ & - & - & - \\
\hline Lying to parents/friends & $\mathrm{X}$ & - & - & - \\
\hline Feeling sad/withdrawn & $\mathrm{X}$ & $\mathrm{X}$ & $\mathrm{X}$ & - \\
\hline Self-harm & $\mathrm{X}$ & $\mathrm{X}$ & $\mathrm{X}$ & - \\
\hline
\end{tabular}

Analysis of participant responses to the 2-part open-ended post-test question "Please write 2 things you see yourself doing to prevent suicide?”, yielded three common themes; (1) talk to them about it, (2) talk to an adult about it and (3) tell them you are there for them. 


\section{Feasibility Analysis}

SWOT. A SWOT analysis was performed to identify the strengths, weaknesses, opportunities and threats of the quality improvement project. Strengths included; low cost, potential for sustainability, evidence-based education and single day implementation.

Weaknesses included; use of a vulnerable population, poor return of consents, no planned follow-up education for reinforcement. Opportunities included; easily duplicated, able to train additional staff for implementation, expand education to additional grades within the same school. Threats included; parental complaints regarding the sensitive nature of subject material and poor participation.

In response to the findings of the SWOT analysis, introduction of the program to parents, school staff members and student body could improve participation in education intervention program. Social determinants in the chosen school district include low education level, low income and poor access to care all potential negative effects on parental consent and participation.

Feasibility and sustainability. The change project was feasible and is sustainable for a several reasons. Ease of access to the recommended population, availability of necessary resources, protocol and short-time intervention all show feasibility of project. The students who were offered participation were already enrolled in the school. The entire protocol was carried out over a 3 week period from initial parental consent to follow-up and appropriate referral for the student who indicated need for further assistance. Beyond the student-led quality improvement project, the school district does not require written consent for participation in an educational program, only opt-out options would be required, thus improving participation rates. 
Sustainability is easily achieved through the continued use of the initial educational program protocol on a yearly basis to students. The initial protocol was for a single grade in a single school; however, the same protocol can become part of standard education curriculum provided by the school. The most important factors in sustainability are adequate implementation and follow-up as needed for students who need access to community resources for mental health care. Additional school staff member(s) may be trained to implement the program improving project sustainability.

Sustainability efforts are currently in the planning phase for implementation of the Signs of Suicide Prevention Program in the coming school year and beyond to the ninth grade students at the original project site. Additional efforts are aiming toward securing funding to broaden use of the program to other grades as well as other area school districts.

\section{Identification of Quality Improvement Project Resources}

Personnel. Minimal personnel were needed for implementation of this quality improvement initiative. Initial resources included the approval and strategic planning with school administrators. I worked with cooperation from the school to obtain parental consent, student assent, and implementation of the suicide prevention education intervention. Additional personnel included the school counselor, who represented available resource professionals if a student disclosed a need to discuss mental health concerns for self or a friend. The additional personnel served an important role in providing adequate and available support services following the educational program for those in need.

Product/services. Signs of Suicide Prevention Program is an education program package that was purchased through a scholarship given by the West Virginia University School of 
Nursing from Screening for Mental Health, Inc. Included in the program package is an adult training video, program implementation manual, student education video, parent/student handouts as well as sample consent forms, student response cards, pre-test, post-test among other additional program printable materials.

Technology. A computer projector was used to present the educational intervention to the participants. Microsoft Excel was utilized for the purposes of organizing data points/test scores. Statistical software was used to evaluate data sets collected in the study.

Budget. Several different components made up the working budget for the change project which is illustrated in the table below.

\begin{tabular}{|l|l|c|}
\hline \multicolumn{2}{|c|}{ ITEM } & \multicolumn{1}{|c|}{ BUDGET } \\
\hline Nigns of Suicide Prevention Program & 1 program kit & TOTAL COST \\
\hline Staff time/DNP student & 6 hours @ 40/hr & $\$ 395.00$ \\
\hline Printed consents/assents/materials & $\begin{array}{l}883 \text { pages @ } \\
0.04 / \text { sheet }\end{array}$ & $\$ 240.00$ \\
\hline Participation Incentive (iTunes Card) & $\begin{array}{l}3 \text { gift cards }(\$ 10.00 \\
\text { value/card) }\end{array}$ & $\$ 30.32$ \\
\hline & & TOTAL $=\$ \mathbf{~ 7 0 0 . 3 2}$ \\
\hline
\end{tabular}

The total cost of the quality improvement project was estimated at: $\$ 700.32$. The majority of this cost was personnel time, which was donated for the proper execution of the education and the evaluation of the effectiveness of the education. The staff time was absorbed by the school officials and staff as part of their pre-designated employment hours. The program materials including the educational videos were part of the cost of the SOS Suicide Prevention Program kit at a cost of 395.00 which was paid for by WVU School of Nursing to aid in project funding. Three iTunes gift cards, each in the amount of $\$ 10.00$ were awarded based on a randomized name drawing following participation in the study which totaled $\$ 30.00$, and were donated. 
Paper products for copies of the pre-test/post-test were covered by the study site, leaving an actual cost of $\$ 0.00$ for project implementation.

\section{Discussion and Recommendations}

Despite the small sample size of the study, results indicate knowledge is gained through the implementation of the Signs of Suicide Prevention Program in the adolescent population. Additional potential application of knowledge gained from the education intervention was identified as a result of the descriptive data collected and analyzed. The three main themes present in the individual descriptive data analysis are consistent with the acronym used for the basis of the educational intervention; ACT: Acknowledge, Care, Tell. This is closely aligned with students most reported responses of (1) talk to them about it, (2) tell them that you are there for them and (3) talk to an adult about it.

Previous studies performed with the use of the SOS Program collected data 3 months following the intervention related to reported behaviors. The current project; however, aimed for further insight and understanding of potential application of knowledge gained through the collection of both individual and group descriptive data. Individual descriptive data remained consistent with a majority of responses mirroring those depicted in the educational film. Eight of the 12 participants described their responses as actions they would perform (i.e., "tell that person I care and I'm there for them"). Conversely, 4 of the 12 participants described their responses as actions they would carry out on behalf of themselves (i.e. "I would also get professional help"). Potentially, the perception of the audience of the film may shape the direction (others or self) of responses and, therefore, knowledge and attitudes about depression/suicide. The SOS Program film shows representations of adolescents interacting with other adolescents regarding topics of 
substance abuse, suicide attempts, school violence, and break-ups. Participants may relate themselves in the role of the teen with signs of depression, or that of the friend.

Group discussions led to 10 major themes with many of the same responses given by all 4 groups; however, two were unique responses to a single group, group \#1: (1) skipping school, (2) lying to parents and friends. These two responses offer insight into not only the group discussion, but also to the potential knowledge or experiences by the group members. Because of the modeling of the Social Learning Theory, additional knowledge potential exists solely within this group above the others. Group learning experiences are easily replicated and feasible in a public school setting as opposed to a PCP or pediatrician office. The school-setting can be an effective learning environment for the social aspect of mental health disparity as well as the growth and developmental stage of adolescents and the importance of peer involvement. For this quality improvement project, 3 students were chosen per group to facilitate group discussion and encourage learning from one another.

In conclusion, the statistically significant increase in post-test scores when compared to test scores prior to the educational intervention, the Signs of Suicide Prevention Program can be a feasible and sustainable means to educate adolescents on these concepts and should be considered for yearly addition to the education curriculum. Despite CDC recommendations adolescents are either not routinely being seen by their Primary Care Provider, or are not being properly educated on mental health concerns. The use of the SOS Prevention Program in the public school system allows for focus on preventative efforts as well as education to students and their peers on proper action if faced with a friend who may be or is suicidal. This quality improvement project required minimal funding, implementation time, resources and can be easily expanded and replicated. Despite the small sample size, the potential for knowledge 
application in a group learning setting through the use of the Social Learning Theory was demonstrated in this quality improvement project. This shows the potential benefits of a public school establishing a role in the mental health education and suicide prevention efforts. 
Appendix A: Site Approval Letter

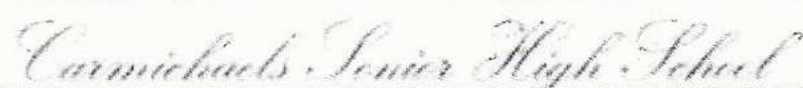

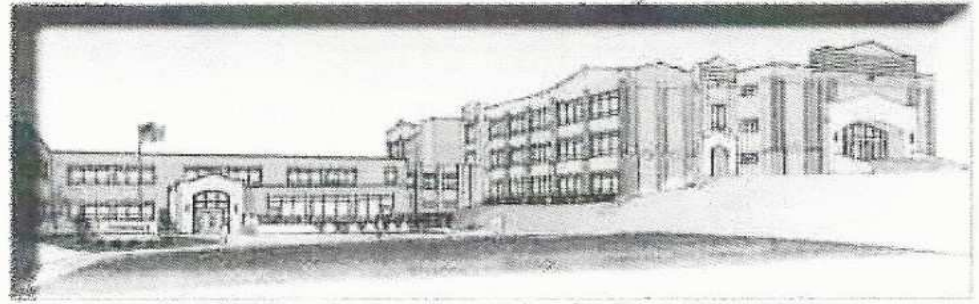

$215 \mathrm{~N}$ Vine Street Carmichaels, PA $15320-1299$

PH: $724 \cdot 966 \cdot 5045$ FAX: 724.966.5556
Www.carmarea.org

\section{Lisa M. Zdravecky - Principal}

Ext. 3106 izdravecky@carmarea.org

September 19, 2016

Mrs. Jessica Tennant,

Congratulations! You have been approved to conduct your Signs of Suicide (SOS) educational program at Carmichaels Senior High School with all of the $9^{\text {th }}$ grade students. Students participating in this program will be required to have written parental consent. With parental consent, you will be able to collect data using a pre and post survey. We look forward to working with you to educate our students about suicide prevention.

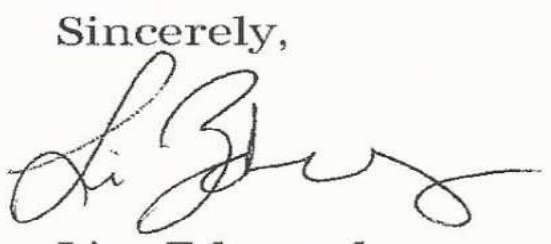

Lisa Zdravecky

Principal 
Appendix B: Parental Consent

\title{
More than Minimal Risk \\ Parental or Guardian Consent Information and HIPAA Form
}

\author{
Principal Investigator Dr. Marilyn Smith \\ Department School of Nursing \\ Protocol Number $\quad 1701410834$ \\ Study Title \\ Implementation of the Signs of Suicide Prevention Program with 9th grade \\ students in a Public School Setting \\ Co-Investigator(s) Jessica Tennant, MSN, CRNP \\ Sponsor (if any)
}

\section{Contact Persons}

In the event your child experiences any side effects or injury related to this research, or if you have any questions, concerns, or complaints about this research you should contact Dr. Marilyn Smith at 304-520-1946. (After hours contact: Dr. Marilyn Smith at 304-520-1946.)

For information regarding your child's rights as a research subject, to discuss problems, concerns, or suggestions related to the research, to obtain information or offer input about the research, contact the Office of Research Integrity \& Compliance (304) 293-7073.

In addition if you would like to discuss problems, concerns, have suggestions related to research, or would like to offer input about the research, contact the Office of Research Integrity and Compliance at 304-293-7073.

\section{Introduction}

Your child, , has been asked to participate in this research study, which has been explained to you by Jessica Tennant, MSN, CRNP. This study is being conducted by Dr. Marilyn Smith in the Department of Nursing at West Virginia University.

\section{Purpose(s) of the Study}

The purpose of this study is to provide suicide prevention education through the implementation of the Signs of Suicide Prevention Program to ninth grade students in a public school setting. The study will examine the knowledge gained by participants about suicide prevention upon completion of the education intervention.

Children in this age group may be at increased risk for depression and/or suicide. Your child has been invited to participate in this research study that involves an evidenced-based educational program which is not part of your child's academic curriculum.

It is expected that this study will enroll approximately 50 subjects to participate in this study.

\section{Description of Procedures}


This study involves receiving education about suicide prevention for a period of up to 35 minutes.

The purpose of the research is to examine the knowledge gained from the education. For this reason, every child who participates in the study will be given the education.

On the day of the study, students with signed parental consents will gather in a classroom in groups no larger than twenty students per group.

Student assent forms (agreements to take part in the study) will be discussed and completed by each student willing to participate in the intervention.

Each participant will receive a 12 -item pre-test which will take approximately 10 minutes to complete.

Students will then view the 22-minute educational DVD.

A brief 10-minute discussion guided on principle concepts of the film using the following scripted question will take place:

a. What kind of changes may you see in a friend that could be a potential sign of depression?

Participants will then be given a 14-item post-test that will take approximately 12 minutes to complete.

Prior to exiting the classroom, each child is able to request a follow-up meeting to discuss any additional concerns through the use of a brief form called a Student Response Card.

Any student who discloses the desire to speak with someone further will be given that opportunity the day the study takes place.

Participation may be terminated by the investigator without regard to the subject's consent which include disruptive behavior in the classroom, observed emotional discomfort or distress.

\section{Risks and Discomforts}

The viewing of the video discussing suicide and suicide prevention in this study may cause emotional stress or distress to participants with sensitive personal or family experiences related to the depictions in the film.

Participants will be permitted to excuse themselves from the study at any time should they chose to do so for any reason. Trained personnel will be available throughout the duration of the study for any participant who may need additional assistance.

\section{Alternatives}

There are no alternatives.

\section{Benefits}


Possible benefits that may result from your child's participation include the increased knowledge related suicide risk and suicide prevention. The knowledge gained from this study may eventually benefit others.

\section{Financial Considerations}

There are no fees for participating in this study.

Each participant of the study will have their name entered for a chance to win an iTunes gift card in the amount of $\$ 10.00$.

\section{Confidentiality}

Any information about your child that is obtained as a result of their participation in this research will be kept as confidential as legally possible. Their research records and test results, just like hospital records, may be subpoenaed by court order or may be inspected by the study sponsor or federal regulatory authorities (including the FDA if applicable) without your additional consent.

In addition, there are certain instances where the researcher is legally required to give information to the appropriate authorities. These would include mandatory reporting of infectious diseases, mandatory reporting of information about behavior that is imminently dangerous to your child or to others, such as suicide, child abuse, etc.

In any publications that result from this research, neither your child's name nor any information from which they might be identified will be published without your consent.

We know that information about your child and their health is private. We are dedicated to protecting the privacy of that information. Because of this promise, we must get your written authorization (permission) before we may use or disclose your child's protected health information or share it with others for research purposes.

A description of this clinical trial will be available on www. ClinicalTrials.gov, as required by US law. This website will not include information that can identify your child. At most, the website will include a summary of the results. You can search this website at any time.

You can decide to sign or not to sign this authorization at the end of this form. However, if you choose not to sign this authorization, your child will not be able to take part in the research study.

\section{HIPAA}

We know that information about your child and their health is private. We are dedicated to protecting the privacy of that information. Because of this promise, we must get your written authorization (permission) before we may use or disclose your child's protected health information or share it with others for research purposes.

You can decide to sign or not to sign this authorization section. However, if you choose not to sign this authorization, your child will not be able to take part in the research study. Whatever choice you make about this research study will not have an effect on your child's access to medical care.

\section{Persons/Organizations Providing the Information}

Your child.

\section{Persons/Organizations Receiving the Information}

- West Virginia University Department of Nursing

- Members and staff of any Institutional Review Board (IRB) that oversees this research study

- West Virginia University Office of Research Integrity and Compliance and Office of Sponsored Programs 


\section{The Following Information Will Be Used}

Responses and scores from pre-test and post-test questionnaires anonymously completed by your child.

\section{The Information is Being Disclosed for the Following Reasons}

An educational requirement of the Doctorate of Nursing Practice program.

Publication of study results (without identifying your child).

\section{You May Cancel this Authorization at Any Time by Writing to the Principal Investigator}

Dr. Marilyn Smith, APRN

WVU School of Nursing

6700 Robert C. Bryd Health Science Center

Morgantown, WV 26506

email: mismith@hsc.wvu.edu

phone: (office) 304-293-2412 (cell) 304-520-1946

fax: 304-293-6826

If you cancel this authorization, any information that was collected already for this study cannot be withdrawn. Once information is disclosed, according to this authorization, the recipient may re-disclose it and then the information may no longer be protected by federal regulations.

This authorization will expire at the end of the study unless you cancel it before that time.

\section{Voluntary Compensation}

If your child is injured as a result of this research, treatment will be available. Responsibility for this treatment will be borne by: the insurance company; OR by you.

In the event that your child is physically injured as a result of participating in this research, care will be available. The Student Assistance Program Coordinator, a mental health professional and members of the Crisis Team will be on site and available if needed. You will be responsible if any further care is needed and will be responsible for the charges of that care. There is no commitment to provide any compensation for research-related injury. You should realize, however, that you have not released this institution from liability for negligence. Please contact the investigator, Dr. Marilyn Smith at (304-520-1946) if you are injured or for further information.

\section{Voluntary Participation}

Refusal to participate or withdrawal will not affect your child's academic standing and will involve no penalty to you.

\section{Signatures}

Upon signing this consent, you will receive a copy.

I willing consent to allow my child to participate in this research. 
Signature of Parent or Guardian

Printed Name

Date

Time

Signature of Investigator or Co-Investigator

Printed Name

Date

Time 
Appendix C: Student Assent

\section{Assent Form}

Principal Investigator

Department

Protocol Number

Study Title

students in a Public School Setting

Co-Investigator(s)

Sponsor (if any)
Dr. Marilyn Smith

School of Nursing

1701410834

Implementation of the Signs of Suicide Prevention Program with 9th grade

Jessica Tennant, MSN, CRNP

\section{Contact Persons}

If you are hurt from being in this research or if you have any questions, concerns, or complaints about this research, you should contact Dr. Marilyn Smith at (304) 520-1946. (After hours contact: Dr. Marilyn Smith at (304) 520-1946).

For information regarding your rights as a person in research or to talk about the research, call the Office of Research Integrity \& Compliance at (304) 293-7073.

\section{Introduction}

You, , have been asked to participate in this research study, which has been explained to you by Jessica Tennant, MSN, CRNP.

\section{Purpose(s) of the Study}

The purpose of this study is to provide suicide prevention education through the implementation of the Signs of Suicide Prevention Program. The study will examine what is learned by participants about suicide prevention after completing the education.

It has been explained to you that children in this age group may be at increased risk for depression and/or suicide. You have been asked to participate in this research study that involves an educational program that is not part of your regular classes. The purpose of this study is to evaluate the effectiveness of the Signs of Suicide Prevention Program in educating ninth grade students about suicide prevention.

\section{Description of Procedures}

This study will be done in place of one of your regularly scheduled classes.

You will be asked to complete a 12 -item TRUE/FALSE test prior to seeing the video that will take about 10 minutes to complete. 
You will then watch a 22-minute DVD.

There will be a 10-minute brief discussion about key concepts of the DVD.

You will then be asked to complete a 14-item test following the discussion that will take about 12 minutes to complete.

Before leaving the classroom you will be able ask to speak to someone any concerns you may have through the use of a brief form called a Student Response Card.

If you ask to talk with someone further, you will be able to do on the same day.

\section{Discomforts}

Some of the situations discussed in the video may be difficult to watch or make you feel sad or upset. You may leave the classroom at any time during the film if needed. Trained adults will be available to you if you need to talk to someone.

\section{Benefits}

This study may help you by learning about the signs of suicide and suicide prevention. This study may also help other people in the future.

\section{Confidentiality}

We promise that anything we learn about you in this study will be kept as secret as possible.

\section{Voluntary Compensation}

If you chose to participate in this study your name will be entered for a chance to win an iTunes gift card in the amount of $\$ 10.00$.

\section{Voluntary Participation}

You do not have to do this. No one will be mad at you if you refuse to do this or if you decide to quit. You have been allowed to ask questions about the research, and all of your questions were answered.

I willingly agree to be in this research.

\section{Signatures}

Signature of Subject

Printed Name

Date

Time

The minor has had the opportunity to have questions addressed. The minor willingly agrees to be in the study. 
Signature of Investigator or Co-Investigator

Printed Name

Date

Time 


\section{Appendix D: Pre-test}

\section{Part I: Pretest: SOS Signs of Suicide Prevention Program}

1. Depression is an illness that doctors can treat.

TRUE

FALSE

2. Most suicide attempts occur without any warning signs or clues.

3. The best thing to tell a suicidal friend is to "pull yourself together and things will get better."

4. People who kill themselves are usually suffering from depression or another mental illness.

5. People who talk about suicide don't really kill themselves.

6. If I talk to someone about their suicidal feelings, it may give them the idea to kill themselves.

7. Drug and/or alcohol abuse is a sign that a person might be depressed and/or suicidal.

8. If someone really wants to kill himself/herself, there is not much anyone can do about it.

9. It's none of my business if a friend says he/she wants to kill himself/herself.

10. Withdrawal from family and friends is a warning sign of depression. 


\section{Appendix E: Post-test}

\section{Part II: Posttest: SOS Signs of Suicide Prevention Program}

1. Depression is an illness that doctors can treat. $\quad$ TRUE FALSE

2. Most suicide attempts occur without any warning signs or clues.

3. The best thing to tell a suicidal friend is to "pull yourself together and things will get better."

4. People who kill themselves are usually suffering from depression or another mental illiness.

6. If I talk to someone about their suicidal feelings, it may give them the idea to kill themselves.

7. Drug and/or alcohol abuse is a sign that a person might be

9. It's none of my business if a friend says he/she wants to kill

10. Withdrawal from family and friends is a warning sign of

12. If a friend tells me they are thinking about killing themselves, I should tell a trusted adult.

Please write 2 things you see yourself doing to prevent suicide?

1.

2. 
Appendix F: Student Response Card

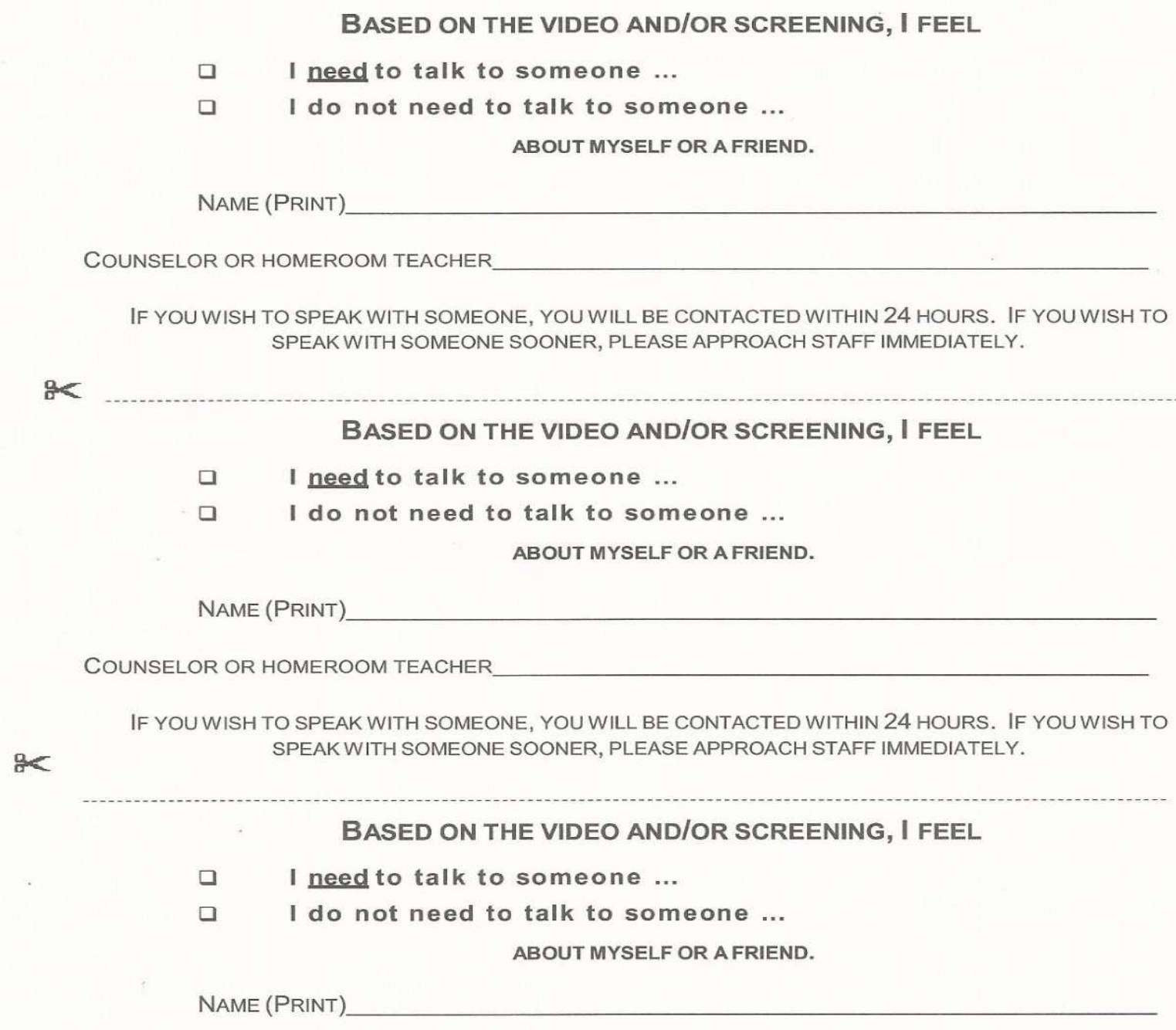

COUNSELOR OR HOMEROOM TEACHER

IF YOU WISTH TO SPEAK WITH SOMEONE, YOU WILL BE CONTACTED WITHIN 24 HOURS. IF YOU WISH TO SPEAK WITH SOMEONE SOONER, PLEASE APPROACH STAFF IMMEDIATELY.

96

BASED ON THE VIDEO AND/OR SCREENING, I FEEL

I I need to talk to someone ...

I do not need to talk to someone ...

ABOUT MYSELF OR A FRIEND.

NAME (PRINT)

COUNSELOR OR HOMEROOM TEACHER

IF YOU WISH TO SPEAK WITH SOMEONE, YOU WILL BE CONTACTED WITHIN 24 HOURS. IF YOU WISH TO SPEAK WITH SOMEONE SOONER, PLEASE APPROACH STAFF IMMEDIATELY. 


\section{References}

Agency for Healthcare Research and Quality. (2015). Depression in children and young people: identification and management in primary, community and secondary care. Retrieved from https://www.guideline.gov/summaries/summary/49118/depression-in-children-and young-people-identification-and-management-in-primary-community-and-secondary care

American Academy of Pediatrics. (n.d.). The Care for Routine Mental Health Screening. Pediatrics. $125: 3$ pp. 133-139

American Psychological Association. (2010). Publication Manual of the American Psychological Association. Washington, D.C.: American Psychological Association.

Anxiety and Depression Association of America. (2016). Anxiety and Depression in Children. Retrieved from https://www.adaa.org/living-with-anxiety/children/anxiety-and $\underline{\text { depression }}$

Aseltine, R.H. \& DeMartino, R. (2004). An Outcome Evaluation of the SOS Suicide Prevention Program. American Journal of Public Health. 94(3). Pp 446-451.

Aseltine, R.H., James, A., Schilling, E.A. \& Glanovsky, J. (2007). Evaluating the SOS suicide prevention program: a replication and extension. BMC Public Health. 7:161.

Bagge, C. L., Glenn, C.R., Lee, H.J. (2013). Quantifying the Impact of Recent Negative Life Events on Suicide Attempts. Journal of Abnormal Psychology. 122(2) pp. 359-368.

Bolognini, B. P., Laget, J., \& Halfon, O. (2003). Adolescent's Suicide Attempts: Populations at Risk, Vulnerability, and Substance Use. Substance Use \& Misuse. 38 (11-13) pp. 16511669. 
The Bureau of Family Health, Division of Child and Adult Health Services. (2014).

Pennsylvania Child Death Annual Report. Retrieved from http://www.health.pa.gov/My $\% 20 \mathrm{Health} /$ Infant $\% 20$ and $\% 20$ Childrens $\% 20 \mathrm{Health} / \mathrm{Newb}$ rns\%20and\%20Infants/Documents/2014_CDR_Annual_Report_FINAL.pdf

Cassels, C. (2009). Majority of US Adolescents with Depression Go Untreated. Medscape. Retrieved from http://www.medscape.com/viewarticle/702869

Carmichaels Area School District. (2016). Mission Statement. Retrieved from http://www.carmarea.org/?DivisionID=18440\&DepartmentID=20541\&ToggleSideNav=

Centers for Disease Control and Prevention. (2016). Suicide Prevention. Retrieved from http://www.cdc.gov/ViolencePrevention/suicide/

Chandra, A. \& Minkovitz, C. (2007). Factors that Affect Mental Health Stigma among $8^{\text {th }}$ Grade Adolescents. J. Youth Adolescents 36:763-774.

HealthyPeople2020. (2016). Mental Health. Retrieved from https://www.healthypeople.gov/2020/leading-health-indicators/2020-lhi-topics/Mental $\underline{\text { Health/determinants }}$

Jacobs, D. (2016). SOS Signs of Suicide High School Prevention Program: Implementation Guide and Resources. USA: Screening for Mental Health

Kennebeck, S. \& Bonin, L. (2016). Suicide behavior in children and adolescents: Epidemiology and risk factors. Retrieved from http://www.uptodate.com/contents/suicidal-behavior-in children-and-adolescents-epidemiology-and-risk-factors 
Learning Theories. (2015). Social Learning Theory (Bandura). Retrieved from https://www.learning-theories.com/social-learning-theory-bandura.html

McLeod, S. (2013). Erik Erikson. Retrieved from http://www.simplypsychology.org/Erik Erikson.html\#identity

Mental Health America. (2016). The State of Mental Health in America. Retrieved from http://www.mentalhealthamerica.net/issues/state-mental-health-america

National Institute of Mental Health. (n.d.) Any Disorder Among Children. Retrieved from http://www.nimh.nih.gov/health/statistics/prevalence/any-disorder-among-children.shtml

NoBullying.com (2015). General Bullying Statistics. Retrieved from https://nobullying.com/general-bullying-statistics/

Schilling, E.A., Aseltine, R.H., James, A. (2015). The SOS Suicide Prevention Program: Further Evidence of Efficacy and Effectiveness. Suicide for Prevention Research. 17:157-166.

U.S. Preventive Services Task Force. (2014). Suicide Risk in Adolescents, Adults and Older Adults. Retrieved from https://www.uspreventiveservicestaskforce.org/Page/Document/RecommendationState $\underline{\text { ntFinal/suicide-risk-in-adolescents-adults-and-older-adults-screening }}$

Weist, M.D., Rubin, M. Moore, E., Adalsheim, S., \& Wrobel, G. (2007). Mental health screening in schools. $J$ Sch Health. 77 (53-58). 